\title{
1,25-Dihydroxyvitamin D3 alleviates salivary adenoid cystic carcinoma progression by suppressing GPX1 expression through the NF-kB pathway
}

\author{
ZHIQUAN HUANG ${ }^{1,2^{*}}$, YEQING LIU ${ }^{1,3^{*}}$, ZIXIAN HUANG ${ }^{1,2}$, HAIFENG LI $^{3}$, \\ XIANGFENG GAN $^{5}$ and ZHUOJIAN SHEN ${ }^{1,5}$
}

${ }^{1}$ Guangdong Provincial Key Laboratory of Malignant Tumor Epigenetics and Gene Regulation,

Sun Yat-sen Memorial Hospital, Sun Yat-sen University, Guangzhou, Guangdong 510120;

Departments of ${ }^{2}$ Oral and Maxillofacial Surgery and ${ }^{3}$ Pathology, Sun Yat-sen Memorial Hospital, Sun Yat-sen University, Guangzhou, Guangdong 510120; ${ }^{4}$ Department of Pathology, The Third Affiliated Hospital, Sun Yat-sen University, Guangzhou, Guangdong 510120; ${ }^{5}$ Department of Thoracic Surgery, Sun Yat-sen Memorial Hospital, Sun Yat-sen University, Guangzhou, Guangdong 510120, P.R. China

Received November 3, 2015; Accepted December 31, 2015

DOI: 10.3892/ijo.2016.3341

\begin{abstract}
Dihydroxyvitamin D3 (1,25D3) is the active form of vitamin D with antineoplastic effects. The glutathione peroxidase-1 (GPX1) gene is associated with tumour progression. The present study aimed to explore the role of GPX1 in 1,25D3-mediated progression of salivary adenoid cystic carcinoma (SACC). Downregulating GPX1 expression inhibited SACC cell proliferation, chemoresistance, motility, and uPA secretion, but promoted apoptosis via the $\mathrm{NF}-\kappa \mathrm{B}$ pathway. Pre-processing 1,25D3 inhibited expression of $\mathrm{NF}-\kappa \mathrm{B} / \mathrm{GPX} 1 / \mathrm{uPA}$, which subsequently suppressed cell motility and cisplatin-resistance in ACC-2 cells. In
\end{abstract}

Correspondence to: Dr Zhiquan Huang, Department of Oral and Maxillofacial Surgery, Sun Yat-sen Memorial Hospital, Sun Yat-sen University, 107 Yanjiang Xi Road, Guangzhou, Guangdong 510120, P.R. China

E-mail: zhiquanhuang1978@126.com

${ }^{*}$ Contributed equally

Abbreviations: 1,25D3, 1,25-dihydroxyvitamin D3; GPX1, glutathione peroxidase-1; GPxs, glutathione peroxidase; ROS, reactive oxygen species; NF- $\mathrm{B}$, nuclear factor-kappa B; SACC, salivary adenoid cystic carcinoma; VDR, vitamin D receptor; PCR, polymerase chain reaction; FBS, fetal bovine serum; CCK-8, Cell Counting kit-8; siRNA, small interfering RNA; ELISA, enzyme linked immunosorbent assay; TBS, Tris-buffered saline; H\&E, haematoxylin and eosin; AA, anhydrous alcohol; NC, negative control; uPA, urokinase-type plasminogen activator; MMP-2, matrix metalloproteinase-2

Key words: vitamin D, glutathione peroxidase-1, nuclear factorkappa B, chemoresistance, cell motility conclusion, 1,25D3 works as a modifier of NF- $\mathrm{BB} / \mathrm{GPX} 1 /$ uPA expression, inhibiting cisplatin-resistance and cell invasive ability of SACC cells. The present study comprehensively elucidated the potential mechanism underlying the effects of vitamin D on chemoresistance and invasive potential in SACC.

\section{Introduction}

Epidemiological studies have indicated that salivary adenoid cystic carcinoma (SACC) is one of the most common types of salivary gland cancers in China, accounting for $11 \%$ of epithelial tumours and $27 \%$ of malignant tumours (1). Clinical data have shown that the low long-term survival rate of SACC is associated with perineural invasion, local recurrence and distant metastasis (2). Chemotherapy is a necessary adjuvant to surgery; however, $\sim 30 \%$ of new cases and $70 \%$ of recurrent cases have platinum-based chemoresistance (3). Thus, there is a growing interest in determining the mechanistic basis for SACC chemoresistance and invasive ability to identify potential therapeutic targets.

GPX1 belongs to the glutathione peroxidase (GPxs) family, the members of which protect the cell membrane from oxidative DNA damage and maintain the body's balance of intracellular redox systems by removing excess reactive oxygen species (ROS) (4). GPX1 modulates many pathophysiologic processes, and overexpression of GPX1 can promote cell invasion, migration and cisplatin resistance in breast, lung, bladder and prostate cancers (5-7). Nuclear factor-kappaB (NF- $\kappa \mathrm{B})$ may regulate GPX1 transcription and expression by combining with the GPX1 promoter region (8-10). However, few studies have reported the tumour-promoting role of GPX1 in head and neck cancers.

1,25-Dihydroxyvitamin $(1,25 \mathrm{D} 3)$ is the active form of vitamin $\mathrm{D}$, which acts as the steroid hormone calcitriol and carries out multiple cellular functions, and 1,25D3 regulates 
Table I. GPX1 siRNA sequences.

\begin{tabular}{llc}
\hline Sequence & \multicolumn{1}{c}{ Forward } & Reverse \\
\hline GPX1 antisense & 5'-GGUACUACUUAUCGAGAAUTT-3' & 5'-AUUCUCGAUAAGUAGUACCTT-3' \\
GPX1 NC & 5'-UUCUCCGAACGUGUCACGUTT-3' & 5'-ACGUGACACGUUCGGAGAATT-3'
\end{tabular}

Table II. Primer sequences for qPCR.

\begin{tabular}{llc}
\hline Gene & \multicolumn{1}{c}{ Forward primer } & Reverse primer \\
\hline GPX1 & 5'-GCGGGGCAAGGTACTACTTA-3' & 5'-CTCTTCGTTCTTGGCGTTCT-3' \\
GADPH & 5'-GCACCGTCAAGGCTGAGAAC-3' & 5'-TGGTGAAGACGCCAGTGGA-3' \\
\hline
\end{tabular}

numerous cellular pathways related to cancer risk and prognosis (11). Clinical studies have suggested that vitamin D deficiency increases the risk of developing cancer and that abundant vitamin D can reduce cancer incidence and improve cancer prognosis and outcome $(12,13)$. Furthermore, it was reported that $1,25 \mathrm{D} 3$ could inhibit $\mathrm{NF}-\kappa \mathrm{B}$ expression in $\mathrm{B}$ lymphocytes, oral squamous cell carcinoma, prostate cancer and melanoma (14-17).

In the preseent study, we first investigated the biological effects of GPX1 on SACC cell lines. Next, we detected whether the NF- $\mathrm{kB}$ pathway was involved in these effects. Finally, we explored whether 1,25D3 alleviates SACC progression by suppressing GPX1 expression through the NF- $\mathrm{KB}$ signalling pathway.

\section{Materials and methods}

The present research was conducted in accordance with the Declaration of Helsinki and the Guide for the Care and Use of Laboratory Animals as adopted and promulgated by the United National Institutes of Health. It has also been approved by the authors' institutional review board.

Cell lines and cell cultures. SACC cell lines (ACC-M, SACC-83 and ACC-2) were purchased from the Cell Bank of the Experimental Animal Center (Sun Yat-Sen University, Guangzhou, China). The cells were cultured in RPMI-1640 medium (Gibco-BRL, Grand Island, NY, USA) supplemented with $10 \%$ fetal bovine serum (FBS) in a $37^{\circ} \mathrm{C}$ humidified incubator containing $5 \% \mathrm{CO}_{2}$.

Reagents and chemicals. The vitamin D metabolite 1,25D3 (Sigma, St. Louis, MO, USA) was dissolved at a concentration of $400 \mu \mathrm{M}$ in anhydrous alcohol (AA) for preservation. Immediately prior to use, the stock was diluted to a final concentration of $30 \mathrm{nM}$ in culture medium. An NF- $\mathrm{KB}$ inhibitor (BAY 11-7082) was purchased from Beyotime Institute of Biotechnology (Shanghai, China).

Small interfering RNA (siRNA) and overexpression vector. The GPX1 overexpression vector, the GPX1 antisense, and the scrambled siRNA were designed and synthesized by Invitrogen (Carlsbad, CA, USA). The GPX1 siRNA sequences used are listed in Table I. Full-length GPX1 coding sequences were PCR-amplified and cloned into a pcDNA3.1 expression vector (Invitrogen) according to the manufacturer's guidelines. DNA sequencing was used to verify the constructs. siRNA transfection was performed using Lipofectamine 2000 reagent (Invitrogen) according to the manufacturer's instructions. Subsequent real-time polymerase chain reaction or western blot analysis was performed to verify changes in GPX1 expression. In the present study, the experimental group was transfected with the antisense GPX1 siRNA or GPX1 overexpression vector, whereas the control group was transfected with a corresponding scrambled sequence.

RNA extraction and PCR. Total RNA was extracted using TRIzol $^{\text {TM }}$ reagent (Sigma-Aldrich, Arklow, Ireland) according to the manufacturer's instructions and was then reverse-transcribed into cDNA using a PrimeScript RT reagent kit (Takara Bio Inc., Shiga, Japan). The newly synthesized cDNA was then used as a template for detection.

Quantitative PCR was carried out using the SYBR-Green method (Takara Bio) in a CFX96 Real-Time PCR Detection system (Bio-Rad Laboratories, Hercules, CA, USA). The primers (Table II) were purchased from Takara Bio, and GAPDH was used as an endogenous control. Amplification was performed according to the manufacturer's protocol (Takara Bio).

Protein extraction and western blot analysis. For protein extraction, cells were washed twice with cold phosphatebuffered saline (PBS), harvested by scraping and lysed in lysis buffer (Beyotime Institute of Biotechnology). Following centrifugation, the supernatant was collected, and protein concentration was determined using a BCA protein assay kit $\left(\right.$ Pierce $^{\mathrm{TM}}$, no. 23227).

For western blotting, $20 \mu \mathrm{l}$ of protein was loaded and separated using $10 \%$ sodium dodecyl sulphate polyacrylamide gel electrophoresis (SDS-PAGE) (Beyotime Institute of Biotechnology); the protein bands were then transferred to Immobilon-P transfer membranes (PVDF) (Beyotime Institute of Biotechnology). The membranes were blocked with 5\% 
non-fat milk in Tris-buffered saline (TBS) containing $0.1 \%$ Tween-20 for $1 \mathrm{~h}$ at room temperature. The blots were probed using antibodies against GPX1 (1:2,000; Abcam), NF- $\kappa$ B P65 (P65, 1:1,000; Cell Signaling Technology, Danvers, MA, USA), phospho-NF-кB P65 (P-p65, 1:1,000; Cell Signaling Technology), urokinase (uPA, 1:1,000; GeneTex, Inc., Irvine, CA, USA) and MMP-2 (1:1,000; GeneTex). Glyceraldehyde 3-phosphate dehydrogenase (GAPDH, 1:2000; Cell Signaling Technology) was used as a loading control. After incubation with a horseradish peroxidase (HRP)-conjugated goat antirabbit immunoglobulin $\mathrm{G}$ secondary antibody, an enhanced chemiluminescence detection method (Pierce ECL Western Blotting Substrate; Thermal Form \& Function, Beverly, MA, USA) was used to visualize the proteins on the blots.

Cell proliferation assay. Cells were plated at a density of $5 \times 10^{3}$ cells/well in 96-well plates, incubated overnight and counted using Cell Counting kit-8 (CCK-8; Dojindo Laboratories, Kumamoto, Japan). The medium in each well was removed, and a mixture of $10 \mu \mathrm{l} \mathrm{CCK-8}$ and $90 \mu \mathrm{l}$ RPMI-1640 medium was added. The plates were incubated for an additional $2.5 \mathrm{~h}$, and absorbance was measured at $450 \mathrm{~nm}$ using a microplate spectrophotometer (Thermo Fisher Scientific, Pittsburgh, PA, USA).

For cisplatin sensitivity testing, cells $\left(7.5 \times 10^{3}\right)$ were seeded into 96-well plates. After overnight incubation, the cells were treated with various concentrations of cisplatin $(0,2.5,5$, 10,20 and $40 \mu \mathrm{mol} / \mathrm{l}$ ), and a CCK-8 assay was performed to examine the cytotoxicity of cisplatin after $48 \mathrm{~h}$ of treatment.

Flow cytometry apoptosis assay. After $24 \mathrm{~h}$ of transfection, cells were plated in 6 -well plates and incubated with $7.5 \mu \mathrm{mol} / 1$ cisplatin for $48 \mathrm{~h}$ at $37^{\circ} \mathrm{C}$. The cells were collected and washed with PBS, and cell apoptosis was analysed by Annexin V/ fluorescein isothiocyanate and propidium iodide staining (Nanjing Keygen Biotech., Co., Ltd., Nanjing, China) using a BD FACSCalibur flow cytometer.

In vitro migration and invasion assays. To assay invasion, $3 \times 10^{5}$ transfected cells were seeded into the upper chamber of a polycarbonate Transwell plate (8-mm pore size; Corning Incorporated, Corning, NY, USA) that was pre-coated with Matrigel (Becton-Dickinson, Bedford, MA, USA). RPMI-1640 containing $20 \%$ FBS was used as a chemoattractant and was added to the lower chamber. After a 24 -h incubation at $37^{\circ} \mathrm{C}$, the cells on the upper surface of the filter were removed. Cells that had traversed the membrane were fixed in methanol at $4^{\circ} \mathrm{C}$ for $20 \mathrm{~min}$ and then stained with $0.1 \%$ crystal violet for $20 \mathrm{~min}$. To microscopically quantify cell migration, the cells were counted in 3 random fields (magnification, x200). To assay migration, a method similar to that used in the invasion assay was employed, with the following modifications: cells were seeded at $1.0 \times 10^{5} /$ chamber into plates with no Matrigel coating and incubated in $600 \mu \mathrm{l}$ RPMI-1640 medium with $10 \%$ FBS in the lower chamber for $18 \mathrm{~h}$.

ELISA assay for $u P A$ and MMP-2. The supernatant of the culture medium of the ACC- 2 cells was collected and centrifuged at $1,000 \times \mathrm{g}$ for $20 \mathrm{~min}$ at room temperature. The concentrations of UPA and MMP-2 in the supernatant were quantified using an uPA ELISA kit (Cloud-Clone Corp., Houston, TX, USA) and an MMP-2 ELISA kit (Shanghai ExCell, Biology, Inc., Shanghai, China) according to the recommended protocols. The detection limits of the assays were $15.6 \mathrm{pg} / \mathrm{ml}(\mathrm{uPA})$ and $0.6 \mathrm{ng} / \mathrm{ml}$ (MMP-2).

In vivo tumorigenicity assay. To explore the effects of 1,25D3 on tumour growth in vivo, ACC- 2 cells were treated for 3 days with $30 \mathrm{nM} 1,25 \mathrm{D} 3$, AA and culture medium in the experimental group, control group and blank group, respectively. Next, 2.0x $10^{6}$ ACC-2 cells were subcutaneously implanted into the right upper backs of $5 \mathrm{BALB} / \mathrm{c}$ nude mice, and the mice were reared for 24 days. The tumours were measured every 3 to 4 days for tumour volume $\left(\mathrm{mm}^{3}\right)$, which equalled length $\mathrm{x}$ width ${ }^{2} \mathrm{x} 0.5$. All mice were subsequently sacrificed, and pieces of tumour tissues were used to establish orthotopic implant models. When tumour volume reached $\sim 50 \mathrm{~mm}^{3}$, $30 \mathrm{nM} 1,25 \mathrm{D} 3, \mathrm{AA}$ and PBS were mixed with $5 \mu \mathrm{M}$ cisplatinum and then intraperitoneally injected into the three groups. Tumour formation was observed and tumour growth curves were constructed. The mice were sactificed 24 days after the injection of the ACC-2 cells, and the tumours were harvested, weighed and frozen or paraffin-embedded. Finally, tissue protein was extracted for analysis.

Immunohistochemistry. Immunohistochemistry was performed on paraffin-embedded tissue sections from tumorigenicity assays. The indicated antibodies (GPX1, 1:50; P65, 1:100; uPA, 1:50; MMP-2, 1:50; and Ki-67, 1:100; GeneTex) were used according to the EnVision HRP detection system (Dako, Carpinteria, CA, USA). After deparaffinization, antigen retrieval was conducted using $10 \mathrm{mM}$ sodium citrate buffer ( $\mathrm{pH} 8.0)$ in a pressure cooker at full power for $5 \mathrm{~min}$. Tissue sections were then treated with $3 \%$ hydrogen peroxide for $10 \mathrm{~min}$. The primary antibodies were diluted with a background-reducing diluent (Dako) according to the manufacturer's specifications and were incubated at $4^{\circ} \mathrm{C}$ overnight. Next, slides were incubated with the EnVision reagent for $30 \mathrm{~min}$ at $37^{\circ} \mathrm{C}$. The slides were then developed with 3,3'-diaminobenzidine for $3 \mathrm{~min}$, counterstained with Meyer's haematoxylin and mounted. The samples were rinsed with phosphate-buffered saline (PBS) between each step.

Statistical analyses. Statistical analyses were performed with SPSS 22.0 software (IBM Inc., Armonk, NY, USA). P-values $<0.05$ were considered to indicate a statistically significant result. Images were created with the Adobe Photoshop CS5 and the GraphPad Prism 5.

\section{Results}

Overexpression of GPX1 promotes cell proliferation, invasion, migration and cisplatin resistance and increases apoptosis in SACC cells. We transfected siGPX1 into 3 SACC cell lines; ACC-2 cells showed relatively effective silence GPX1, with an $80 \%$ GPX1 reduction compared with other cells (Fig. 1A and G). Therefore, ACC-2 cells were selected for further experimentation. When we further transfected cells with GPX1 vector and negative control (NC) sequences, GPX1 increased by $40 \%$. Twenty-four hours after transfection, we performed 

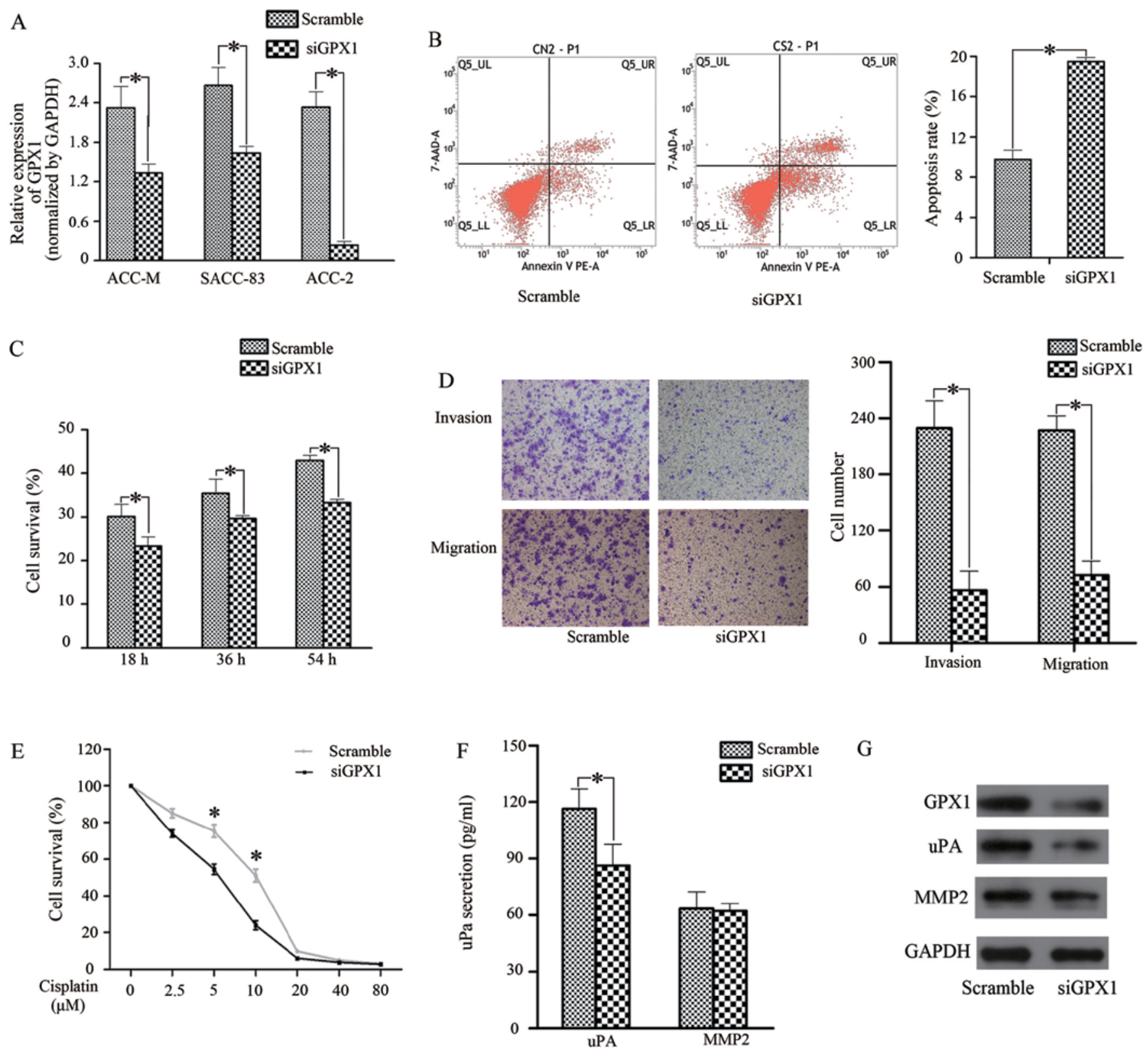

Figure 1. Downregulation of GPX1 suppresses ACC-2 cell biological functions. Expression levels of GPX1 in three SACC cell lines transfected with GPX1 siRNA or scramble sequences were detected using (A) RT-PCR and (G) western blot analysis. Downregulation of GPX1 reduces (C) ACC-2 cells proliferation, but (B) promotes apoptosis and (E) cisplatin sensitivity. Morphologic comparison of cells penetrating the artificial basement membrane (magnification, x200) (D); the results show that repression of GPX1 expression significantly inhibited the invasion and migration of ACC-2 cells. (G) Western blotting and (F) ELISA demonstrated that GPX1 induces the secretion and activation of uPA. ${ }^{*} \mathrm{P}<0.05$ compared with the ACC-2 scramble group.

CCK-8 assays to detect the effect of GPX1 on ACC-2 cell proliferation at three time-points (18,36 and $54 \mathrm{~h})$. Cell proliferative capacity was reduced in the siGPX1 group (Fig. 1C). As for cisplatin resistance, as the expression of GPX1 decreased, the cells displayed the same trend, particularly at concentrations of 5 and $10 \mu \mathrm{M}$ (Fig. 1E). Next, we selected a $5 \mu \mathrm{M}$ concentration of cisplatin for use in a flow cytometry apoptosis assay and found that the apoptosis rate in the siGPX1 cells (19.50\%) was higher than that in the control (9.74\%) (Fig. 1B). Next, a Transwell assay was performed; as shown in Fig. 1D, siGPX1 cells displayed weak invasive and migratory abilities. In contrast, upregulation of GPX1 promoted proliferation (Fig. 2A), cisplatin resistance (Fig. 2C), invasion and migration
(Fig. 2D) but decreased apoptosis (Fig. 2B) in ACC-2 cells. Taken together, these results indicated that GPX1 overexpression in ACC-2 cells could be responsible for enhanced cell proliferation, cisplatin resistance, invasion and migration.

GPX1-enhanced invasion and migration is associated with $u P A$. We speculated that the contribution of GPX1 to SACC motility might involve the downstream factors MMP-2 or uPA $(18,19)$. At $24 \mathrm{~h}$ after transfection, the expression and secretion of uPA and MMP-2 were tested (Figs. 1F and G, and $2 \mathrm{E}$ and $\mathrm{F})$. The results showed that $\mathrm{UPA}$ secretion was dramatically reduced, but MMP-2 remained stabile when GPX1 was reduced. Meanwhile, uPA secretion increased when cells were 

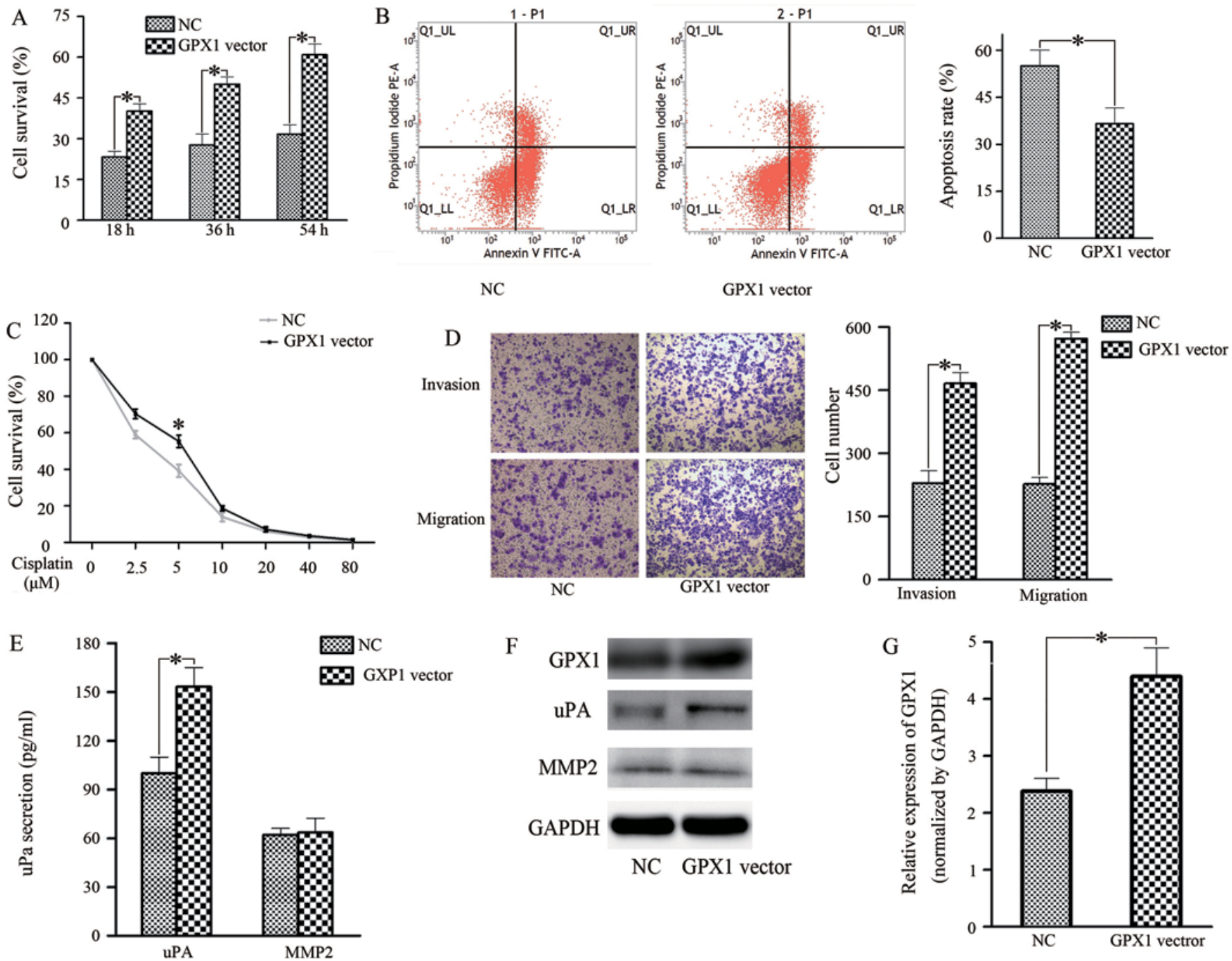

Figure 2. Upregulation of GPX1 promotes ACC cell biological functions. The ACC-2 cell line was transfected with the GPX1 vector, and the expression of GPX1 was detected using (G) RT-PCR and (F) western blot analysis. Upregulation of GPX1 promotes cell proliferation (A), cisplatin resistance (C), invasion and migration (D, magnification, x200) but decreases cell apoptosis (B). Furthermore, uPA secretion was increased in ACC-2 cells transfected with the GPX1 vector, as detected by (F) western blotting and (E) ELISA. " $\mathrm{P}<0.05$ compared with the ACC-2 negative control (NC) group.

transfected with the GPX1 overexpression vector. Based on these results, we considered that GPX1-enhanced invasion and metastasis in ACC-2 cells is associated with uPA.

The NF- $\kappa B$ pathway is related to GPX1-mediated SACC biological effects. To clarify the mechanism by which GPX1 exerts its effects, we investigated the role of the NF- $\kappa B$ pathway (20). Twenty-four hours after treatment with BAY 11-7082, western blotting showed that NF- $\mathrm{\kappa B}$ (P65) expression was significantly downregulated (Fig. 3E). Cell proliferation (Fig. 3A), cisplatin resistance (Fig. 3B), and invasive and migratory ability (Fig. 3D) were all reduced; correspondingly, apoptosis increased when the concentration of cisplatin reached $5 \mu \mathrm{M}$ (Fig. 3C). GPX1 expression and uPA expression were downregulated, but MMP-2 expression was sustained (Fig. 3E). Next, we performed ELISA to detect uPA and MMP-2 secretion. The results showed that UPA secretion was reduced, but MMP-2 showed no change (Fig. 3F). These findings suggested that the NF- $\kappa B$ pathway was related to proliferation, cisplatin resistance, invasion, migration and apoptosis in SACC via positively regulating GPX1 expression and UPA activation.

1,25D3 regulates $S A C C$ cell biological effects through the $N F-\kappa B$ pathway. We further investigated the biological role of $1,25 \mathrm{D} 3$ in SACC. After preprocessing with 1,25D3 for 3 days, the proliferative capacity (Fig. 4A) and cisplatin resistance (Fig. 4C) of ACC-2 cells were reduced. As for their invasive and migratory capacities, cells treated with 1,25D3 displayed weaker motility compared to controls (Fig. 4D). Cell apoptosis assays showed no significant difference (Fig. 4B) after 1,25D3 treatment. These results illustrated that $1,25 \mathrm{D} 3$ was able to reduce cell proliferation, cisplatin resistance and motility in ACC-2 cells.

We next examined if the reduced biological function of 1,25D3-treated ACC-2s was related to activation of NF- $\kappa \mathrm{B}$ signalling and subsequent downregulation of GPX1 expression. To test this hypothesis, we examined NF- $\kappa \mathrm{B}$ and GPX1 expression in ACC-2 cells after 1,25D3 treatment. As anticipated, NF- $\mathrm{kB}, \mathrm{GPX} 1$ and uPA expression was inhibited 
A

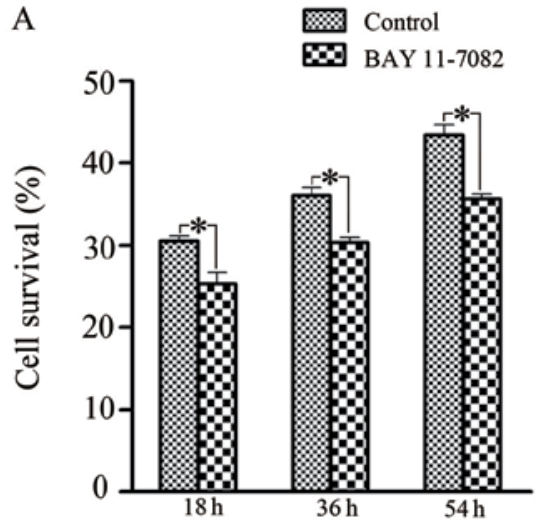

$\mathrm{C}$

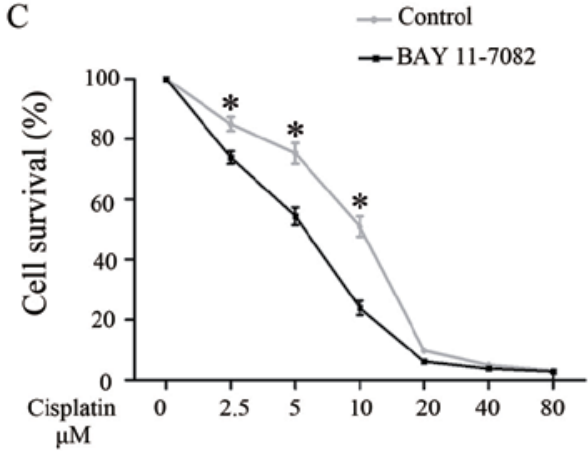

$\mathrm{E}$

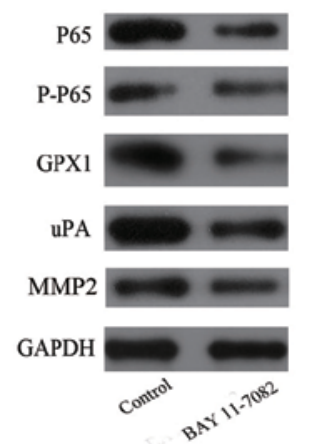

B
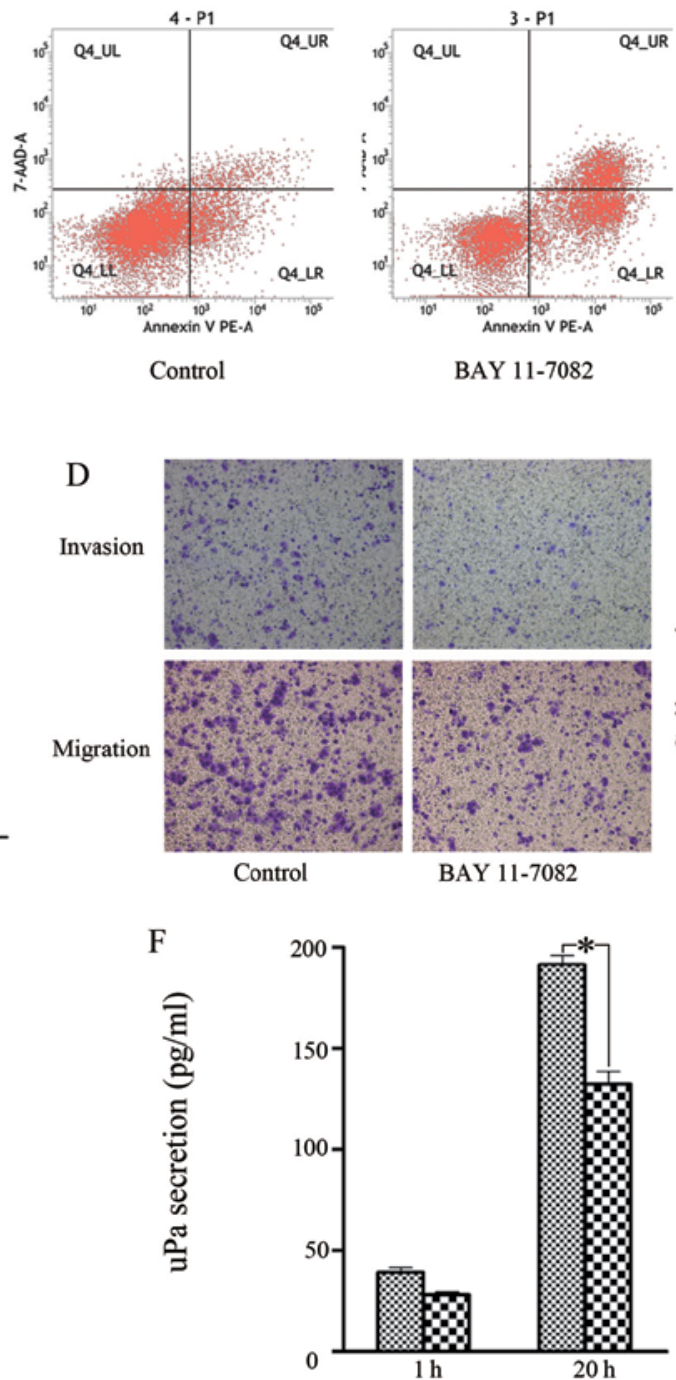
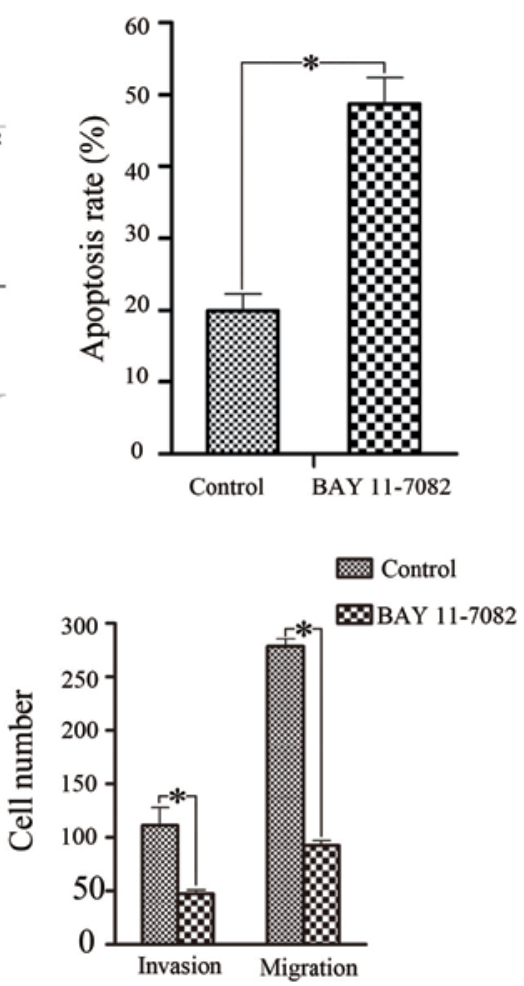

Control

BAY 11-7082

Figure 3. The NF-кB pathway is involved in GPX1-regulated cellular biological functions in ACC-2 cells. After cells were treated with BAY 11-7082, the expression of NF- $\mathrm{kB}$ (P65) was inhibited (E). ACC-2 cells treated with BAY 11-7082 reduced proliferation (A), cisplatin resistance (C), invasion and migration (D, magnification, x200), whereas apoptosis (B) was increased. The expression of GPX1 (E) and uPA (E and F) were inhibited. "P<0.05 compared with ACC-2 control group.

(Fig. 4E). Moreover, ELISA results showed that uPA secretion was reduced in 1,25D3-treated ACC-2 cells, whereas MMP-2 showed no change (Fig. 4F). Taken together, these results indicate that $1,25 \mathrm{D} 3$ may inhibit GPX1 expression to regulate biological functions in SACC cells through the $\mathrm{NF}-\kappa \mathrm{B}$ pathway.

In vivo, 1,25D3 alleviates SACC progression by inhibiting GPX1 expression through the $N F-\kappa B$ pathway. To further explore the potential function and mechanism of 1,25D3 in vivo, we performed tumorigenicity assays. On day 24 , the mean tumour weight of the experimental group was lower than those of the two control groups (Fig. 5A). According to the tumour growth curves (Fig. 5B), obvious tumour nodules formed in the control and blank groups on day 4 after injection with ACC-2 cells, whereas in the experimental group this occurred on day 5 . The surfaces of the tumours in the experimental group began to visibly fester on day 18 after injection with
ACC-2 cells, and the tumours stopped growing. In contrast, tumours began to visibly fester on day 20 in the control groups, and the tumours continued to grow until day 24 . H\&E staining of the tumours revealed that the experimental group had more significant necrotic areas than the control groups (Fig. 5D). Furthermore, the expression of GPX1, P65, P-P65 and uPA in the experimental group was lower than that in the control and blank groups (Fig. 5C). Additionally, immunohistochemical analysis of the inoculated tumours revealed that the tumours of the experimental group expressed significantly lower levels of Ki-67, uPA and P65, with no change in MMP-2 expression and increased GPX1 expression (exclusive cytoplasmic expression; no nuclear expression) (Fig. 5E).

\section{Discussion}

SACC is a malignant tumour arising from secretory epithelial cells in the salivary glands of the head and neck. The 5-year 
A

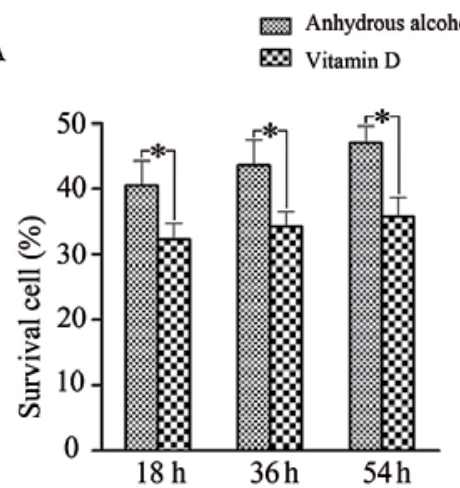

C

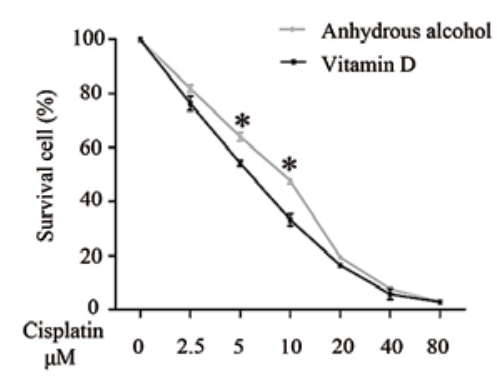

E

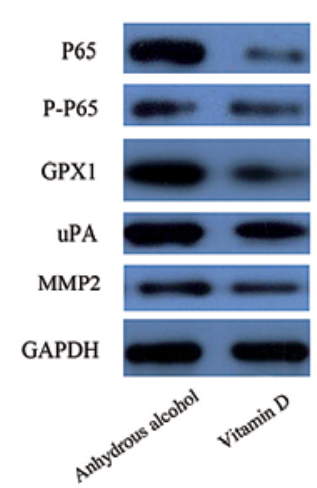

B
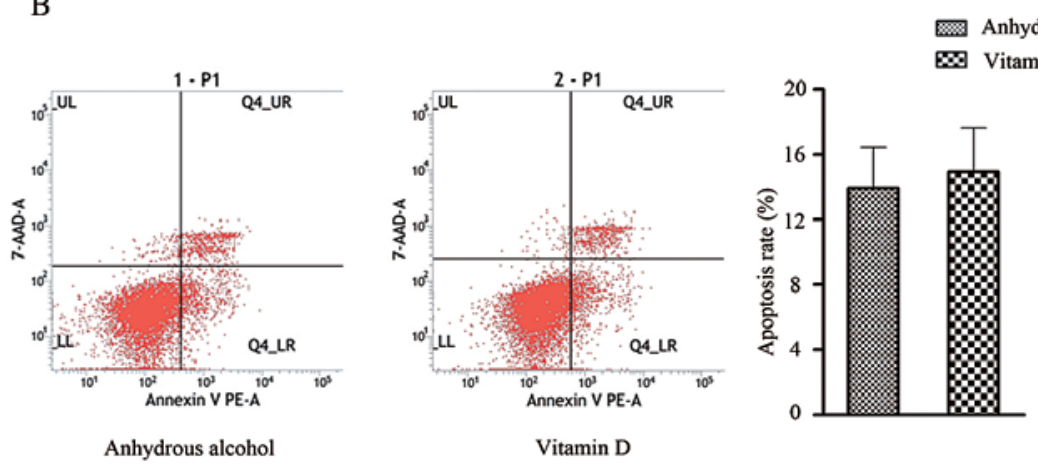

D
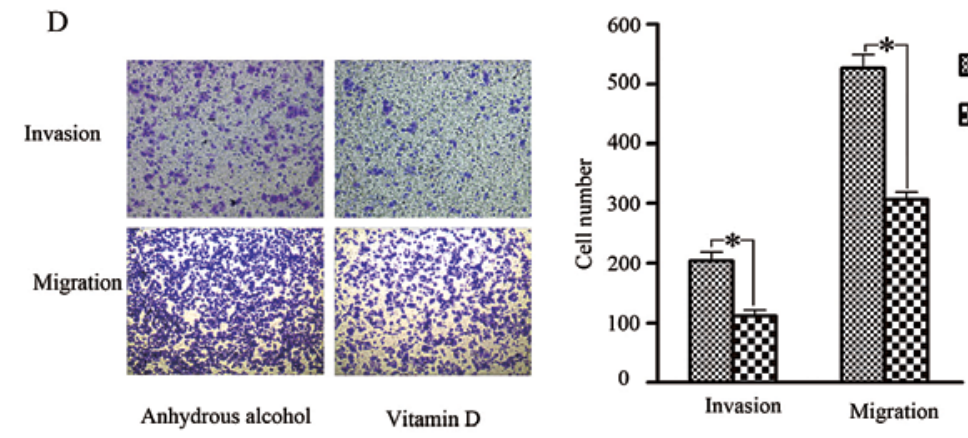

F

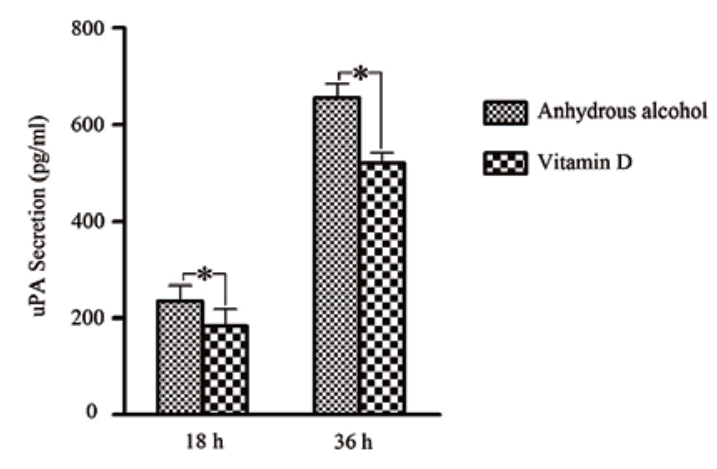

Figure 4. 1,25VD3 regulates ACC progression by suppressing GPX1 expression through the NF- $\mathrm{B}$ pathway in vitro. After treatment with 1,25VD3, (A) ACC-2 cell proliferative capacity and $(\mathrm{C})$ cisplatin resistance were reduced in comparison with the control groups, whereas no significant difference in cell apoptosis (B). 1,25VD3 reduced cell invasion and migration of ACC-2 cells (D, magnification, $x 200)$, nor the expressions of GPX1 and NF- $\kappa$ B (P65) (E). ELISA also showed that $1,25 \mathrm{VD} 3$ reduces the secretion and activation of $\mathrm{uPA}(\mathrm{F}) .{ }^{*} \mathrm{P}<0.05$ compared with the ACC-2 anhydrous alcohol (AA) group.

disease-free survival rate is $\leq 90 \%$; however, the survival rate is reduced to $40 \%$ after 15 years (2). SACC has a poor prognosis, primarily owing to its insidious invasion into adjacent tissues and haematogenous spread to distant organs (lungs, bone and liver) (21-23). Therefore, it is necessary to identify and understand the mechanism behind SACC chemoresistance and metastasis to improve treatment strategies for SACC patients.

Recent studies have found that upregulation of GPx expression leads cancer cells to develop drug resistance by reducing ROS produced by platinum-based chemotherapy drugs, which promotes tumourigenesis $(24,25)$. In the present study, we found that GPX1 acts as a key factor in regulating SACC progression, as GPX1 suppression reduced ACC-2 cell proliferation, cisplatin resistance and motility while increasing cell apoptosis. Upregulation of GPX1 shows the corresponding opposite effect. It has been reported that the generation of MMP-2 and UPA promotes tumour invasion and metastasis in squamous cell carcinoma of the head and neck $(18,19)$. Following GPX1 downregulation, we detected a reduction of uPA expression and secretion in ACC-2 cells, whereas MMP-2 remained stable. In contrast, uPA secretion and activation increased when GPX1 was overexpressed.

In malignant tumours, $\mathrm{NF}-\kappa \mathrm{B}$ expression can regulate downstream protein expression, thereby regulating tumour chemoresistance and invasion $(8,26-28)$. In the present study inhibiting the $\mathrm{NF}-\kappa \mathrm{B}$ pathway reduced $\mathrm{ACC}-2$ cell growth, cisplatin resistance, and motility, while promoting apoptosis. To further analyse the role of NF- $\kappa \mathrm{B}$-regulated factors, the expression of GPX1 was decreased, and uPA secretion and expression showed the same trend. Thus, we established a correlation between $\mathrm{NF}-\kappa \mathrm{B}$ and GPX1 in ACC- 2 cells and 
A
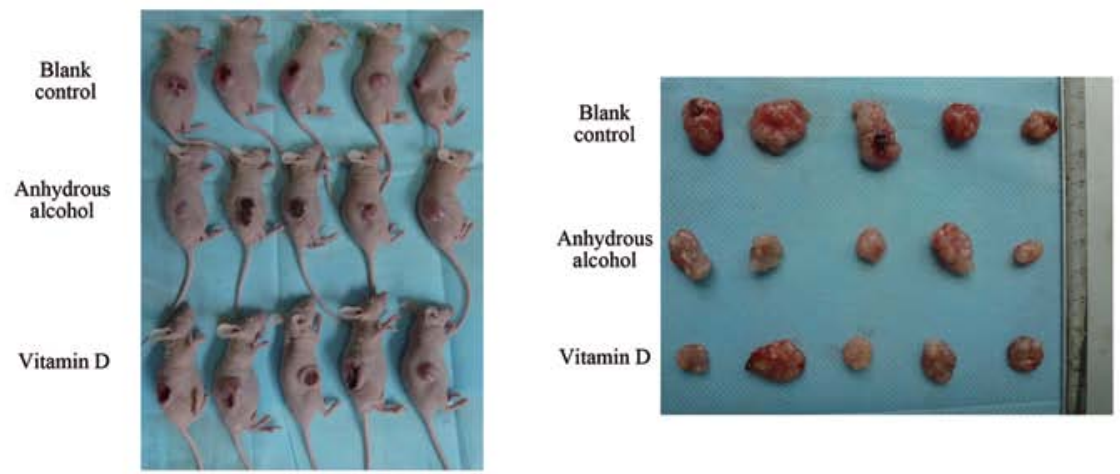
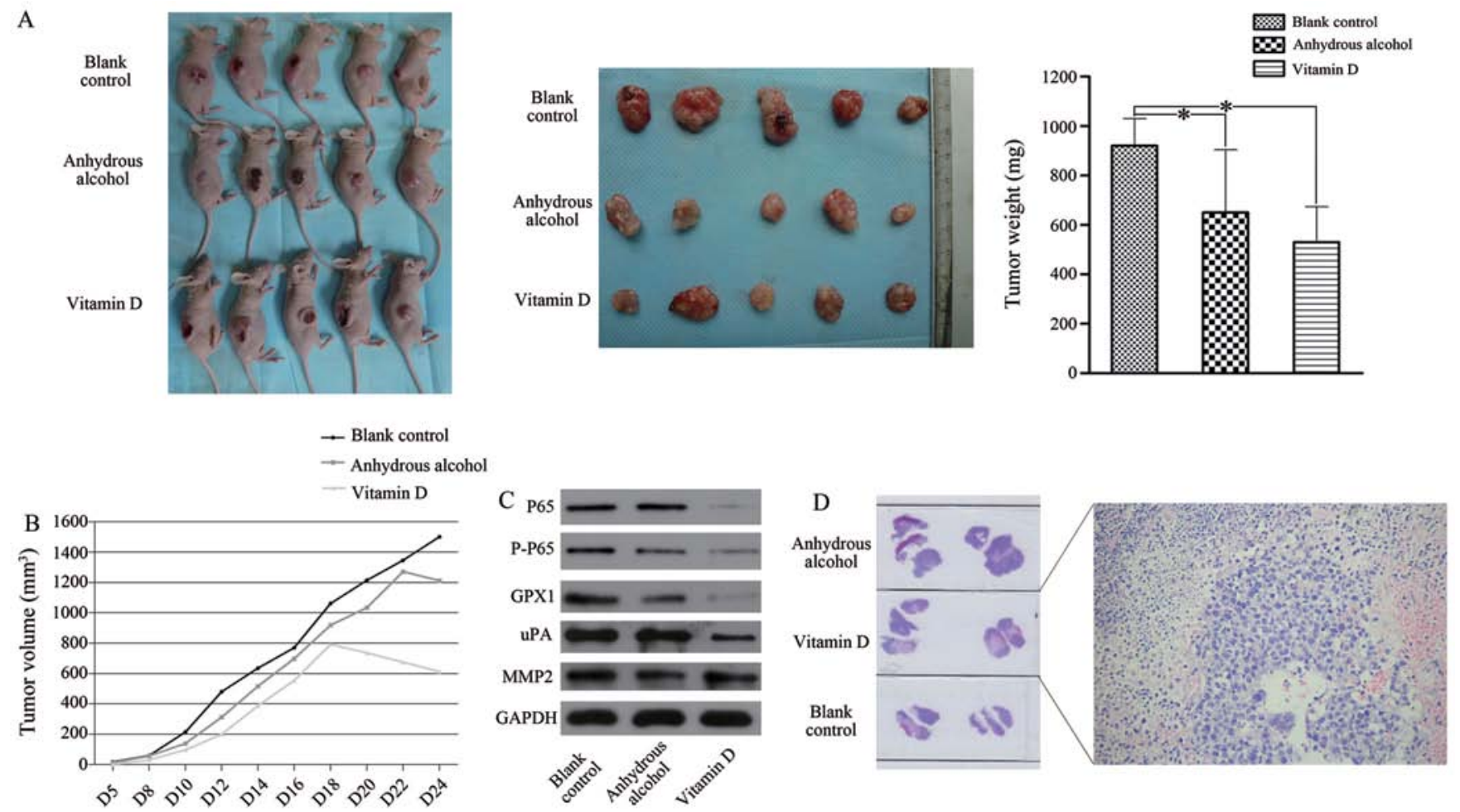

E

Ki67

P65

GPX1
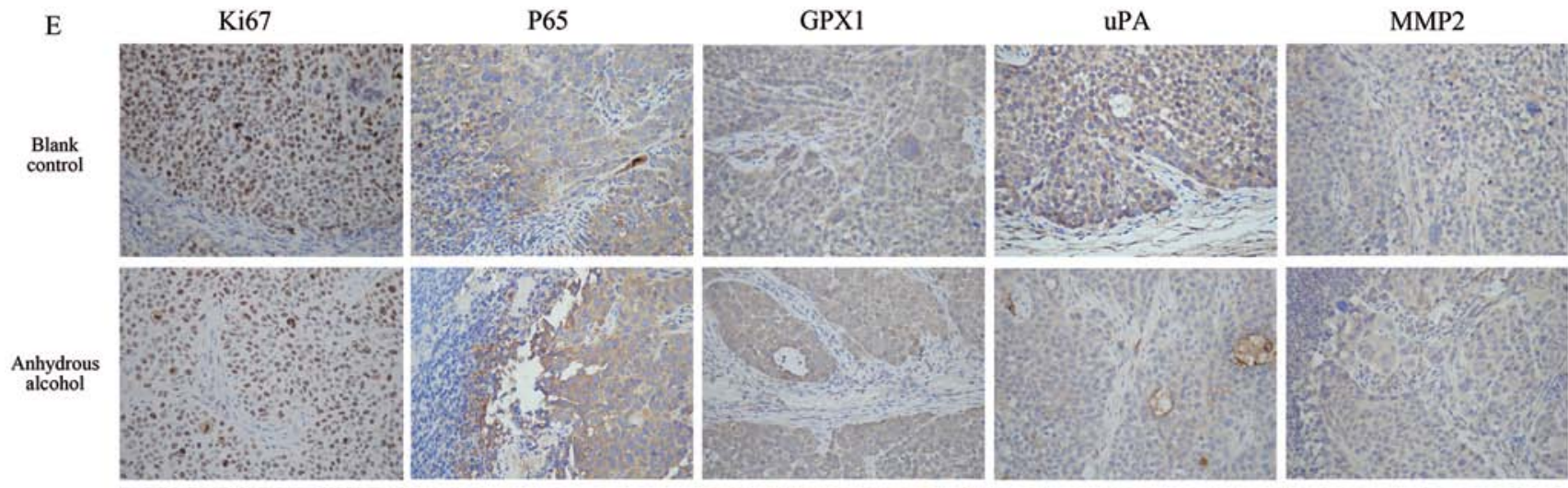

Vitamin D
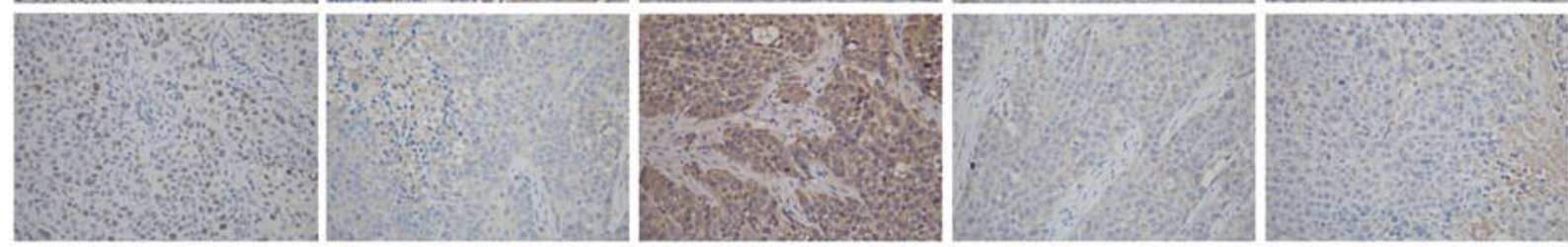

Figure 5. 1,25VD3 reduces the malignancy of ACC-2 cells in vivo. (A) The volume and weight of the transplanted tumours were significantly decreased in the vitamin D. (B) The tumour growth curve showed that vitamin D group had the slowest growth rate, and tissue necrosis appeared from the 18th day. H\&Estained slices of transplanted tumours formed by ACC-2 cells also suggested that 1,25VD3 promoted cell necrosis (D, magnification, x200). The expression levels of GPX1, P65, P-P65 and uPA were significantly decreased in vitamin D group compared with the other two control groups (C). Immunohistochemistry staining indicated that the levels of P65, uPA and Ki-67 were downregulated in vitamin D group. No difference in MMP- 2 expression was noted among these groups. GPX1 showed only cytoplasmic staining and was highly expressed in the experimental group (E, magnification, $\mathrm{x} 200)$. "P<0.05 compared with the blank control group.

confirmed that GPX1-induced uPA secretion is mediated by an NF-kB-dependent pathway, which modulates SACC chemoresistance and invasion.

Vitamin D has widespread actions throughout the human body, and supplementation may be a strategy for preventing cancer incidence and/or tumour progression $(29,30)$. Mechanistically it has been suggested that vitamin $\mathrm{D}$ regulates a wide range of factors, including interleukin- 8 , NF- $\mathrm{KB}$, HBp17 and miR98, which subsequently inhibit tumour development $(12,14-17,31,32)$. As expected, we found that the active metabolite of vitamin D, 1,25D3, inhibited ACC- 2 cell proliferation, cisplatin resistance, invasion and migration and alleviated SACC progression in vivo. We analysed the mechanism by which $1,25 \mathrm{D} 3$ reduced the extent of SACC malignancy. After treatment with 1,25D3, NF- $\mathrm{KB}$ and GPX1 activation were responsively decreased in ACC-2 cells, and the secretion and expression of uPA were also reduced. Tumorigenicity assays confirmed that pre-applied 1,25D3 could inhibit the progres- 
sion of SACC tumours; subsequent immunohistochemical examination also detected signal alterations among $\mathrm{NF}-\kappa \mathrm{B}$, GPX1 and uPA. Our combined in vitro and in vivo experiments revealed that GPX1 is a target of 1,25D3, which alleviates SACC progression by suppressing GPX1 expression through the $\mathrm{NF}-\kappa \mathrm{B}$ pathway. This provides a theoretical basis for vitamin $\mathrm{D}$ supplementation in cancer management. Avoiding vitamin D deficiency and adding supplements may be an economical and effective way to reduce cancer incidence and improve cancer prognosis.

In conclusion, in the present study, we demonstrated that downregulation of GPX1 can suppress SACC cell proliferation, cisplatin-resistance, migration, and invasion and promote apoptosis through the NF- $\mathrm{B}$ pathway and uPA activation. $1,25 \mathrm{D} 3$ achieved its antineoplastic function via the abovementioned regulators. Collectively, establishing 1,25D3 as a modifier of $\mathrm{NF}-\kappa \mathrm{B} / \mathrm{GPX} 1 / \mathrm{uPA}$ expression provides a novel therapeutic strategy for the treatment of SACC.

\section{Acknowledgements}

The present study was supported by grants from the Key Laboratory of Malignant Tumor Molecular and Translational Medicine of Guangzhou Bureau of Science and Information Technology (no. [2013]163), the Key Laboratory of Malignant Tumor Gene Regulation and Target Therapy of Guangdong Higher Education Institutes (no. KLB09001). It was also supported by the National Natural Science Foundation of China (no. 81101592), the Fundamental Research Funds for the Central Universities (no. 13ykpy26) and the Guangdong Province Natural Science Foundation (no. S2013010014794).

\section{References}

1. Thompson L: World Health Organization classification of tumours: Pathology and genetics of head and neck tumours. Ear Nose Throat J 85: 74, 2006.

2. Fordice J, Kershaw C, El-Naggar A and Goepfert H: Adenoid cystic carcinoma of the head and neck: Predictors of morbidity and mortality. Arch Otolaryngol Head Neck Surg 125: 149-152, 1999.

3. Colevas AD: Chemotherapy options for patients with metastatic or recurrent squamous cell carcinoma of the head and neck. J Clin Oncol 24: 2644-2652, 2006.

4. Brigelius-Flohé R: Tissue-specific functions of individual glutathione peroxidases. Free Radic Biol Med 27: 951-965, 1999

5. Lubos E, Loscalzo J and Handy DE: Glutathione peroxidase-1 in health and disease: From molecular mechanisms to therapeutic opportunities. Antioxid Redox Signal 15: 1957-1997, 2011.

6. Fu TY, Hou YY, Chu ST, Liu CF, Huang CH, Chen HC, Hsiao M, Lu PJ, Wang JS and Ger LP: Manganese superoxide dismutase and glutathione peroxidase as prognostic markers in patients with buccal mucosal squamous cell carcinomas. Head Neck 33: 16061615, 2011.

7. Schumacker PT: Reactive oxygen species in cancer cells: Live by the sword, die by the sword. Cancer Cell 10: 175-176, 2006

8. Gong G, Méplan C, Gautrey H, Hall J and Hesketh JE: Differential effects of selenium and knock-down of glutathione peroxidases on $\mathrm{TNF} \alpha$ and flagellin inflammatory responses in gut epithelial cells. Genes Nutr 7: 167-178, 2012.

9. Schreck R, Albermann K and Baeuerle PA: Nuclear factor kappa B: An oxidative stress-responsive transcription factor of eukaryotic cells (Review). Free Radic Res Commun 17: 221-237, 1992.

10. Vibet S, Goupille C, Bougnoux P, Steghens JP, Goré J and Mahéo K: Sensitization by docosahexaenoic acid (DHA) of breast cancer cells to anthracyclines through loss of glutathione peroxidase (GPx1) response. Free Radic Biol Med 44: 1483-1491, 2008.

11. Feldman D, Krishnan AV, Swami S, Giovannucci E and Feldman BJ: The role of vitamin D in reducing cancer risk and progression. Nat Rev Cancer 14: 342-357, 2014.
12. Ting HJ, Messing J, Yasmin-Karim S and Lee YF: Identification of microRNA-98 as a therapeutic target inhibiting prostate cancer growth and a biomarker induced by vitamin D. J Biol Chem 288: $1-9,2013$.

13. Krishnan AV, Swami S and Feldman D: The potential therapeutic benefits of vitamin $\mathrm{D}$ in the treatment of estrogen receptor positive breast cancer. Steroids 77: 1107-1112, 2012.

14. Bao BY, Yao J and Lee YF: 1alpha, 25-dihydroxyvitamin D3 suppresses interleukin-8-mediated prostate cancer cell angiogenesis. Carcinogenesis 27: 1883-1893, 2006.

15. Tse AK, Zhu GY, Wan CK, Shen XL, Yu ZL and Fong WF: 1alpha,25-Dihydroxyvitamin D3 inhibits transcriptional potential of nuclear factor kappa B in breast cancer cells. Mol Immunol 47: $1728-1738,2010$

16. Rosli SN, Shintani T, Hayashido Y, Toratani S, Usui E and Okamoto T: $1 \alpha, 25 \mathrm{OH} 2 \mathrm{D} 3$ down-regulates HBp17/FGFBP-1 expression via NF- $\mathrm{KB}$ pathway. J Steroid Biochem Mol Biol 136: 98-101, 2013.

17. Janjetovic Z, Brozyna AA, Tuckey RC, Kim TK, Nguyen MN, Jozwicki W, Pfeffer SR, Pfeffer LM and Slominski AT: High basal NF- $\kappa \mathrm{B}$ activity in nonpigmented melanoma cells is associated with an enhanced sensitivity to vitamin D3 derivatives. Br J Cancer 105: 1874-1884, 2011.

18. Huang Z, Huang H, Li H, Chen W and Pan C: EMMPRIN expression in tongue squamous cell carcinoma. J Oral Pathol Med 38: 518-523, 2009.

19. Huang Z, Tan N, Guo W, Wang L, Li H, Zhang T, Liu X, Xu Q, Li J and Guo Z: Overexpression of EMMPRIN isoform 2 is associated with head and neck cancer metastasis. PLoS One 9: e91596, 2014

20. Ghashghaeinia M, Toulany M, Saki M,Bobbala D, Fehrenbacher B, Rupec R, Rodemann HP, Ghoreschi K, Röcken M, Schaller M, et al: The NFkB pathway inhibitors Bay 11-7082 and parthenolide induce programmed cell death in anucleated erythrocytes. Cell Physiol Biochem 27: 45-54, 2011.

21. Kim KH, Sung MW, Chung PS, Rhee CS, Park CI and Kim WH: Adenoid cystic carcinoma of the head and neck. Arch Otolaryngol Head Neck Surg 120: 721-726, 1994.

22. Matsuba HM, Spector GJ, Thawley SE, Simpson JR, Mauney M and Pikul FJ: Adenoid cystic salivary gland carcinoma. A histopathologic review of treatment failure patterns. Cancer 57: 519-524, 1986.

23. Sung MW, Kim KH, Kim JW, Min YG, Seong WJ, Roh JL, Lee SJ, Kwon TK and Park SW: Clinicopathologic predictors and impact of distant metastasis from adenoid cystic carcinoma of the head and neck. Arch Otolaryngol Head Neck Surg 129: 1193-1197, 2003

24. Takata Y, Kristal AR, Santella RM, King IB, Duggan DJ, Lampe JW, Rayman MP, Blount PL, Reid BJ, Vaughan TL, et al: Selenium, selenoenzymes, oxidative stress and risk of neoplastic progression from Barrett's esophagus: Results from biomarkers and genetic variants. PLoS One 7: e38612, 2012.

25. Cao M, Mu X, Jiang C, Yang G, Chen H and Xue W: Singlenucleotide polymorphisms of GPX1 and MnSOD and susceptibility to bladder cancer: A systematic review and metaanalysis. Tumour Biol 35: 759-764, 2014.

26. Dibra D, Mishra L and Li S: Molecular mechanisms of oncogeneinduced inflammation and inflammation-sustained oncogene activation in gastrointestinal tumors: An under-appreciated symbiotic relationship. Biochim Biophys Acta 1846: 152-160, 2014.

27. Harte MT, Gorski JJ, Savage KI, Purcell JW, Barros EM, Burn PM, McFarlane C, Mullan PB, Kennedy RD, Perkins ND, et $a l$ : NF- $\kappa \mathrm{B}$ is a critical mediator of BRCA1-induced chemoresistance. Oncogene 33: 713-723, 2014.

28. Wang H, Khor TO, Yang Q, Huang Y, Wu TY, Saw CL, Lin W, Androulakis IP and Kong AN: Pharmacokinetics and pharmacodynamics of phase II drug metabolizing/antioxidant enzymes gene response by anticancer agent sulforaphane in rat lymphocytes. Mol Pharm 9: 2819-2827, 2012.

29. Murphy AB, Nyame Y, Martin IK, Catalona WJ, Hollowell CM, Nadler RB, Kozlowski JM, Perry KT, Kajdacsy-Balla A and Kittles R: Vitamin D deficiency predicts prostate biopsy outcomes. Clin Cancer Res 20: 2289-2299, 2014.

30. Grant WB: Vitamin D status: Ready for guiding prostate cancer diagnosis and treatment? Clin Cancer Res 20: 2241-2243, 2014.

31. Gilmore TD: The Rel/NF-kappaB signal transduction pathway: Introduction. Oncogene 18: 6842-6844, 1999.

32. Thota C, Laknaur A, Farmer T, Ladson G, Al-Hendy A and Ismail $\mathrm{N}$ : Vitamin $\mathrm{D}$ regulates contractile profile in human uterine myometrial cells via NF- $\kappa \mathrm{B}$ pathway. Am J Obstet Gynecol 210: 347.e1-347.e10, 2014. 